\title{
Linear and nonlinear heart-rate analysis in a rat model of acute anoxia
}

\author{
Hernâni Gonçalves ${ }^{1}$, Tiago Henriques-Coelho ${ }^{2}$, João Bernardes ${ }^{3,4}$, \\ Ana Paula Rocha ${ }^{1,5}$, Ana Nogueira ${ }^{2}$ and Adelino Leite-Moreira ${ }^{2}$ \\ ${ }^{1}$ Departamento de Matemática Aplicada, Faculdade de Ciências, Universidade do Porto, \\ Rua do Campo Alegre 687, 4169-007 Porto, Portugal \\ ${ }^{2}$ Serviço de Fisiologia, Faculdade de Medicina, Universidade do Porto, Alameda Prof. Hernâni \\ Monteiro, 4200-319 Porto, Portugal \\ ${ }^{3}$ Departamento de Ginecologia e Obstetrícia, Faculdade de Medicina, Universidade do Porto, \\ Alameda Prof. Hernâni Monteiro, 4200-319 Porto, Portugal \\ ${ }^{4}$ Instituto Nacional de Engenharia Biomédica (INEB), Porto, Portugal \\ ${ }^{5}$ Centro de Matemática da Universidade do Porto, Porto, Portugal \\ E-mail: hernani.goncalves@fc.up.pt and joaobern@med.up.pt
}

Received 4 July 2008, accepted for publication 25 July 2008

Published 10 September 2008

Online at stacks.iop.org/PM/29/1133

\begin{abstract}
The objective of this study was the assessment of heart-rate (HR) dynamics with linear and nonlinear methods during episodes of mechanical ventilation and acute anoxia in rats. Namely, to assess whether linear and nonlinear HR analysis was able to discriminate acute anoxia from baseline in rats and if this was consistent with human foetal and adult studies. Five HR segments of 1 min duration, during baseline recording, mechanical ventilation and first, second and third minutes of induced acute anoxia, were analysed in ten adult Wistar rats. Linear time and frequency domain and nonlinear methods were used, namely mean HR (mHR), long-term irregularity (LTI), interval index (II), low frequency (LF) and high frequency (HF), approximate entropy (ApEn) and sample entropy (SampEn). New parameters for the entropy indices are proposed for the analysis of rats' HR. Bootstrap percentile confidence intervals and nonparametric statistical tests were used in the evaluation of the differences between segments. During mechanical ventilation a clear spectral band was detectable at the ventilation rate, but $\mathrm{mHR}$, II and the 'new' entropy indices were the only significantly changed indices. In the transition from baseline-mechanical-ventilation to mechanical-ventilationinduced anoxia, a statistically significant decrease of mHR, II and entropy indices was observed, clearly discriminating these two instances, whereas most linear indices increased. With continued anoxia, most linear indices decreased significantly, whereas entropy remained stably low. These results are consistent with other foetal human and non-human studies and evidence that the rat model may be used for further research on linear and nonlinear analysis of heart-rate dynamics. The transition from baseline to acute anoxia was encompassed by
\end{abstract}


signs of increased activation of the autonomic nervous system sympathetic branch, and decreased or blunted activity of the HR complexity regulatory centres.

Keywords: heart rate, variability, linear analysis, entropy, anoxia, Wistar rats

\section{Introduction}

Heart-rate (HR) analysis remains one of the most important clinical and research tools in medicine. This is particularly true regarding foetal heart-rate (FHR) analysis, the most widely used method of foetal surveillance in western industrialized countries (ACOG 2005). In this field, but also in adult medicine, several nonlinear and linear time and frequency domain indices have been developed for HR analysis (Task Force 1996, Beckers et al 2006a, Richman and Moorman 2000). However, many questions remain to be answered before the use of these HR indices can be advised in clinical practice. It is already known that these indices are influenced by equipment, signal acquisition mode, sampling rate and pre-processing, but studies about these issues are still limited (Task Force 1996, Gonçalves et al 2006a). In the same way, some studies have contributed to elucidate the mathematical and pathophysiological meaning of these indices, as well as their clinical validity, but many doubts still persist about these matters (Gonçalves et al 2006b, Gonçalves et al 2007). We have performed some studies using linear and nonlinear HR analysis in human foetuses, both before and during labour (Gonçalves et al 2006a, 2006b, 2007). However, studies on human subjects have always inherent difficulties, particularly during foetal life.

The Wistar rat model has been used for heart-rate analysis research by some centres (Moraru et al 2004, Murasato et al 1998), and it is a model particularly well known at our institution in cardiovascular research (Henriques-Coelho et al 2004, Lourenço et al 2006). Moraru et al (2004) have studied linear and nonlinear HR dynamics in rats recovering from an episode of cardiac arrest. The same research group has also studied linear and nonlinear HR dynamics regarding the recovery from anoxia of rats pre-conditioned to hypoxia (Moraru et al 2005). In any case, linear and nonlinear HR analysis provided useful and discriminative information regarding the physiopathological monitoring of the studied conditions.

The objective of this study is the assessment of HR analysis with linear and nonlinear methods, with a focus on episodes of mechanical ventilation and acute anoxia in rats, much in the same way as it occurs to the foetus during a complicated labour. Namely, with this study we intended to assess whether linear and nonlinear HR analysis was able to discriminate acute anoxia from baseline in rats and if this was consistent with human foetal and adult studies. The ultimate goal is a better understanding of the meaning of these methods, regarding their application in clinical research and practice, namely in the field of computerized FHR analysis (Bernardes et al 1991, 1998, Ayres-de-Campos et al 2000, 2005).

\section{Methods}

\subsection{Animal model}

Animal experiments were performed according to the Portuguese law for animal welfare and conform to the National Institutes of Health Guide for the Care and Use of Laboratory 
Animals (NIH Pub. No. 85-23, Revised 1996). Ten adult female Wistar rats (Charles River Laboratories, Barcelona, Spain) weighing 250-290 g were housed in groups of five rats/cage in a controlled environment under a 12:12-h light-dark cycle at a room temperature of $22^{\circ} \mathrm{C}$, with a free supply of food and water.

Once a week for 5 weeks, the rats were anaesthetized with ketamin $\left(50 \mathrm{mg} \mathrm{kg}^{-1}\right)$ and ECG monitored for $20 \mathrm{~min}$. In the last week, after $20 \mathrm{~min}$ of acquisition, they were also submitted to mechanical ventilation, with a respiratory frequency of $1.25 \mathrm{~Hz}$ and volume of $2 \mathrm{~mL}$, during $1 \mathrm{~min}$ and followed by $5 \mathrm{~min}$ of induced anoxia, with ventilation being $\mathrm{CO}_{2} 5 \%$ and $\mathrm{N}_{2} 95 \%$.

\subsection{Heart-rate acquisition and pre-processing}

Four ECG electrodes were placed subcutaneously, one in each rat leg, according to a standardized procedure. The ECG signal was acquired at a sampling rate of $500 \mathrm{~Hz}$, and the tachogram was obtained after automatic R wave detection in the ECG signal and posterior medical validation. Subsequently, the tachogram was converted to a heart-rate (HR) signal in bpm.

The sampling rate of $500 \mathrm{~Hz}$ was assumed as a good methodological compromise, after preliminary tests with sampling rates of $1000 \mathrm{~Hz}$, as well as parabola adjustment around the $500 \mathrm{~Hz}$ ECG R wave, which did not significantly change our analysis. In particular, we used integer values of HR signal (in bpm), as it occurs in most foetal heart-rate (FHR) acquisition equipment. Therefore, the $500 \mathrm{~Hz}$ ECG sampling rate is sufficient to achieve an HR signal with relatively good accuracy.

The HR signal was then resampled at a frequency rate of $8 \mathrm{~Hz}$ using cubic spline interpolation, in order to ensure the correct coverage of the considered spectral bands (described in the next section).

\subsection{Linear and nonlinear heart-rate analysis}

Five 1-min segments without identified signal loss were analysed: during baseline recording, during mechanical ventilation and during the first three minutes of induced anoxia. An example of a full HR signal and its respective five segments is presented in figure 1 . The spikes visible in figure 1 correspond to ectopic beats, which were not eliminated since they may represent a 'typical' pattern of anoxia periods - rather than isolated points present in normal recordings of HRV (animal or adult) - avoiding the loss of important information present in the signal.

The analysis included common time- and frequency-domain measures, as previously described in detail (Gonçalves et al 2006a). The time-domain measures included mean HR (mHR), HR standard deviation (sdHR), long-term irregularity (LTI), the mean amplitude between the maximum and minimum for each minute $(\Delta)$, short-term variability (STV) and interval index (II).

For spectral characterization of the data, nonparametric spectrum estimation was performed (Welch method, Hanning window, 256-point segment, 62.5\% of superposition) (Hayes 1996). Several spectral bands are referred to in the literature, regarding spectral analysis of HR in rats (Kuwahara et al 1994, Cerutti et al 1991). The decomposition of HR variability allowed the identification of a very low frequency component (VLF), below $0.04 \mathrm{~Hz}$, and two components associated with the low and high frequencies (LF and HF), centred approximately at $0.1 \mathrm{~Hz}(0.04-1.00 \mathrm{~Hz})$ and at the respiratory frequency $(1.00-3.00 \mathrm{~Hz})$, respectively (Kuwahara et al 1994). It is currently believed that HF is related to the 


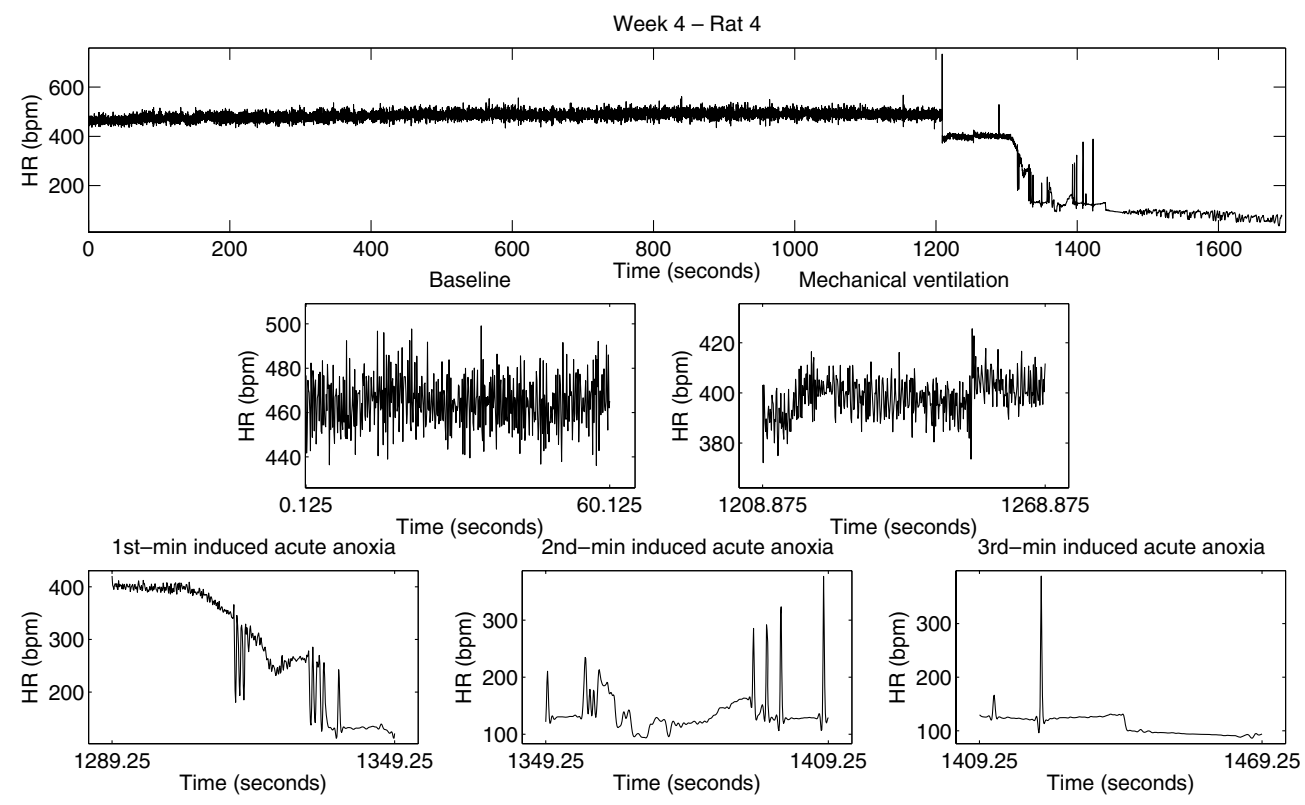

Figure 1. Typical heart-rate record of a normal anaesthetized rat, during 20 baseline minutes, followed by $1 \mathrm{~min}$ of mechanical ventilation at $1.25 \mathrm{~Hz}$ and $5 \mathrm{~min}$ of induced anoxia (at the top), and the five 1-min segments extracted for the study analysis.

parasympathetic system, while the LF is associated also with the sympathetic activity (Task Force 1996).

The nonlinear indices considered in this study, ApEn and SampEn, which have broadly been applied in human heart-rate signal analysis, had also been shown to be useful as a measure of regularity in experiments on rats (Pincus et al 1996), although with much less application in this field. For approximate entropy (Pincus and Viscarello 1992), ApEn $(m, r)$, and Sample Entropy (Richman and Moorman 2000), $\operatorname{SampEn}(m, r)$, which are nonlinear measures obtained through direct signal estimation, capable of quantifying signal complexity (or irregularity) robustly with short segments, the parameter $m$ is the embedding dimension and the parameter $r$ acts as a threshold. The considered estimator for $\operatorname{ApEn}(m, r)$, based on $N$ signal points, was

$$
\begin{aligned}
\operatorname{ApEn}(m, r, N) & =\phi^{m}(r)-\phi^{m+1}(r) \\
& =\frac{1}{N-m+1} \sum_{i=1}^{N-m+1} \ln \left(C_{r}^{m}(i)\right)-\frac{1}{N-m} \sum_{i=1}^{N-m} \ln \left(C_{r}^{m+1}(i)\right)
\end{aligned}
$$

SampEn was computed in a similar manner as ApEn, with the major difference that SampEn does not count self-matches, having reduced bias when compared to ApEn. The values $0.1 S D, 0.15 S D$ and $0.2 S D$ are typically considered for the parameter $r$, and the value 2 for parameter $m$, regarding foetal heart-rate analysis (Pincus and Viscarello 1992). These values for $r$ and $m$ produce good statistical validity of $\operatorname{ApEn}(m, r)$, based on the calculations that included both theoretical analysis and clinical applications (Pincus 1995). However, these parameters may not be most appropriate for the HR analysis of rats. The ratio between the mean coefficient of variation of FHR signals and the mean coefficient of variation of HR signals of (normal) rats is roughly about 6. Although ApEn and SampEn are normalized by 

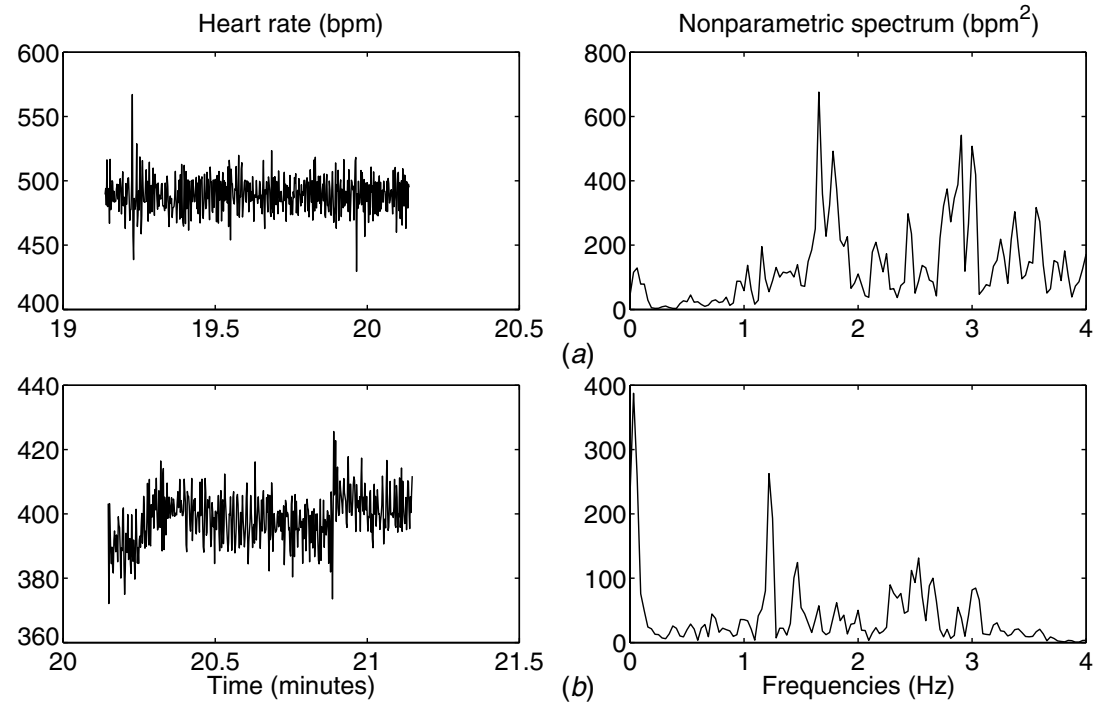

Figure 2. The heart-rate signal of the minutes before (a) and during mechanical ventilation at $1.25 \mathrm{~Hz}$ (b) and their correspondent nonparametric spectrum. A clear spectral component at $1.25 \mathrm{~Hz}$ corresponding to the mechanical ventilation is visible.

the standard deviation, and since the variation around the HR baseline in rats is about six times less, we consider $10 \%, 15 \%$ or $20 \%$ (using parameters $0.1,0.15$ and 0.2 ) of a much lesser variation around the HR baseline. Therefore, we propose the values $0.6 S D, 0.9 S D$ and 1.2SD for the parameter $r$ of ApEn and SampEn, regarding the analysis of HR signals of rats. The segment length, $N$, considered for ApEn and SampEn computation, was equal to the number of points on each 1-min analysed segment (480 points). For both ApEn and SampEn measures, higher complexity leads to higher entropy values.

For statistical inference, bootstrap percentile $95 \%$ confidence intervals for the median (Martinez and Martinez 2002) were computed for the five considered segments: baseline, mechanical ventilation, and the first three minutes of the anoxia period. The differences between these five segments were evaluated through a nonparametric Mann-Whitney statistical test (Dudewicz and Mishra 1988).

\section{Results}

Table 1 presents bootstrap percentile $95 \%$ confidence intervals for linear and nonlinear indices calculated over the five 1-min segments, during baseline recording, mechanical ventilation and during the first, second and third minutes of acute anoxia.

During mechanical ventilation a clear spectral band was detectable at the ventilation rate of $1.25 \mathrm{~Hz}$, as shown in figure 2. However, we did not find statistical significance in the sequence baseline-mechanical-ventilation regarding frequency indices (table 1). This apparent contradiction may be easily justified by the fact that the $1.25 \mathrm{~Hz}$ spectral component is quite narrow (figure 2), giving little contribution to the frequency indices (in particular to the HF spectral band and the LF/HF index), since spectral indices are based on the calculation of areas below the spectrum. 
Table 1. Bootstrap percentile $95 \%$ confidence intervals for the median of linear and nonlinear $(m=2)$ indices calculated over 1-min segments, during baseline recording, mechanical ventilation and the first, second and third minutes of induced acute anoxia. For further explanations and abbreviations see text. (a) $p<0.05$ baseline versus mechanical ventilation; (b) $p<0.05$ baseline versus anoxia (1st min); (c) $p<0.05$ mechanical ventilation versus anoxia (1st min); (d) anoxia (1st min) versus anoxia (2nd $\mathrm{min}$ ); (e) anoxia (2nd min) versus anoxia (3rd min).

\begin{tabular}{|c|c|c|c|c|c|c|}
\hline $\begin{array}{l}\text { Heart-rate } \\
\text { indices }\end{array}$ & Baseline & $\begin{array}{l}\text { Mechanical } \\
\text { ventilation }\end{array}$ & $\begin{array}{l}\text { Anoxia } \\
(1 \text { st } \mathrm{min})\end{array}$ & $\begin{array}{l}\text { Anoxia } \\
(2 n d \min )\end{array}$ & $\begin{array}{l}\text { Anoxia } \\
\text { (3rd min) }\end{array}$ & $p$ \\
\hline $\mathrm{mHR}$ & $464-492$ & $391-432$ & $284-307$ & $138-200$ & $112-150$ & $\mathrm{a}, \mathrm{b}, \mathrm{c}, \mathrm{d}$ \\
\hline sdHR & $8.1-11.7$ & $7.5-16.8$ & $51.5-97.5$ & $31.8-53.9$ & $16.9-42.1$ & $\mathrm{~b}, \mathrm{c}, \mathrm{d}$ \\
\hline LTI & $10.1-14.2$ & $11.8-24.4$ & $108.8-241.9$ & $34.6-159.3$ & $31.0-58.7$ & $\mathrm{~b}, \mathrm{c}$ \\
\hline$\Delta$ & $49-75$ & $56-142$ & $307-682$ & $123.5-283$ & $130-303$ & $\mathrm{~b}, \mathrm{c}, \mathrm{d}$ \\
\hline STV & $7.7-11.2$ & $3.9-13.1$ & $17.4-22.0$ & $8.9-23.3$ & $4.2-28.9$ & $\mathrm{~b}, \mathrm{c}$ \\
\hline II & $0.93-1.18$ & $0.46-0.90$ & $0.22-0.37$ & $0.20-0.69$ & $0.51-0.66$ & $\mathrm{a}, \mathrm{b}, \mathrm{c}$ \\
\hline $\mathrm{TP}$ & $60-119$ & 26-234 & 994-1607 & $142-722$ & 241-1229 & $\mathrm{b}, \mathrm{c}$ \\
\hline LF & $7.3-20.1$ & $3.0-16.6$ & $376.2-745.3$ & $78.3-425.8$ & $207.5-864.8$ & $\mathrm{~b}, \mathrm{c}$ \\
\hline $\mathrm{HF}$ & $30.6-74.5$ & $17.7-176.9$ & $238.1-545.4$ & $1.3-67.5$ & $23.0-694.50$ & $\mathrm{~b}$ \\
\hline $\mathrm{LF} / \mathrm{HF}$ & $0.11-0.53$ & $0.09-0.32$ & $1.37-2.94$ & $6.31-74.06$ & $2.52-13.48$ & $\mathrm{~b}, \mathrm{c}, \mathrm{d}$ \\
\hline $\operatorname{ApEn}(0.1)$ & $0.74-0.77$ & $0.77-0.98$ & $0.31-0.58$ & $0.29-0.43$ & $0.23-0.66$ & $\mathrm{a}, \mathrm{b}, \mathrm{c}$ \\
\hline $\operatorname{ApEn}(0.15)$ & $1.07-1.11$ & $1.09-1.13$ & $0.24-0.48$ & $0.20-0.36$ & $0.21-0.63$ & $\mathrm{~b}, \mathrm{c}$ \\
\hline $\operatorname{ApEn}(0.2)$ & $1.23-1.33$ & $1.13-1.29$ & $0.19-0.41$ & $0.14-0.31$ & $0.19-0.58$ & $\mathrm{~b}, \mathrm{c}$ \\
\hline $\operatorname{SampEn}(0.1)$ & $1.93-2.45$ & $1.64-2.35$ & $0.20-0.50$ & $0.20-0.36$ & $0.10-0.68$ & $\mathrm{~b}, \mathrm{c}$ \\
\hline $\operatorname{SampEn}(0.15)$ & $1.75-2.18$ & $1.48-2.08$ & $0.12-0.32$ & $0.14-0.27$ & $0.09-0.58$ & $\mathrm{~b}, \mathrm{c}$ \\
\hline $\operatorname{SampEn}(0.2)$ & $1.71-2.05$ & $1.33-1.94$ & $0.08-0.23$ & $0.10-0.22$ & $0.10-0.52$ & $\mathrm{~b}, \mathrm{c}$ \\
\hline $\operatorname{ApEn}(0.6)$ & $1.10-1.17$ & $0.65-1.09$ & $0.06-0.20$ & $0.03-0.17$ & $0.11-0.32$ & $a, b, c$ \\
\hline $\operatorname{ApEn}(0.9)$ & $0.75-0.82$ & $0.41-0.72$ & $0.04-0.13$ & $0.02-0.14$ & $0.09-0.25$ & $a, b, c$ \\
\hline $\operatorname{ApEn}(1.2)$ & $0.52-0.54$ & $0.26-0.50$ & $0.02-0.12$ & $0.01-0.12$ & $0.06-0.18$ & $\mathrm{a}, \mathrm{b}, \mathrm{c}$ \\
\hline $\operatorname{SampEn}(0.6)$ & $1.04-1.15$ & $0.50-1.05$ & $0.03-0.12$ & $0.03-0.07$ & $0.04-0.25$ & $a, b, c$ \\
\hline $\operatorname{SampEn}(0.9)$ & $0.65-0.73$ & $0.26-0.65$ & $0.02-0.08$ & $0.02-0.06$ & $0.03-0.17$ & $a, b, c$ \\
\hline $\operatorname{SampEn}(1.2)$ & $0.45-0.48$ & $0.17-0.45$ & $0.01-0.06$ & $0.01-0.05$ & $0.03-0.12$ & $a, b, c$ \\
\hline
\end{tabular}

Also from figure 2, an ultra low frequency component is noticeable, which we found in some rats for some segments (without any systematic pattern of appearance) and may reflect small fluctuations of the baseline, which are only reflected in the very low frequencies (not considered for analysis).

In the sequence baseline-mechanical-ventilation, mHR, II, as well as most entropy indices, changed to significantly lower values. Before and during acute anoxia, statistically significant differences were found for the majority of the computed indices. The sequence mechanical-ventilation-induced anoxia led to a decrease of mean HR and entropy, clearly discriminating these two instances, whereas most linear indices increased. With continued anoxia, most linear indices decreased, whereas entropy remained stably low. Figures 3-5 illustrate the evolution of indices $m H R, L F$ and $\operatorname{SampEn}(2,1.2)$ during the five different periods, for each recording.

A numerical experiment was performed to evaluate if the entropy of the second and third minutes of induced acute anoxia could reflect the variability of the interpolation method rather than the real HR fluctuations. The results of using linear interpolation instead of cubic spline interpolation did not significantly change the results that had been achieved. In particular, the entropy indices were the less susceptible of being affected by the interpolation method. 


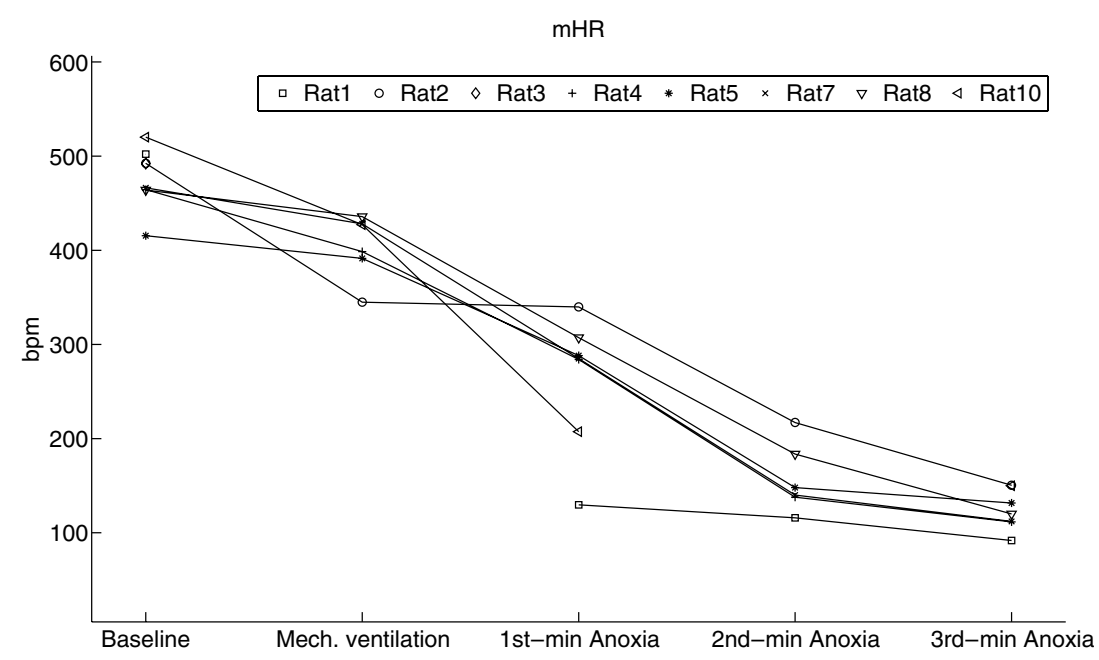

Figure 3. Mean heart rate (mHR) calculated for each rat recording, over 1-min segments without signal loss, in the sequence baseline-mechanical-ventilation-induced anoxia (first, second and third minutes).

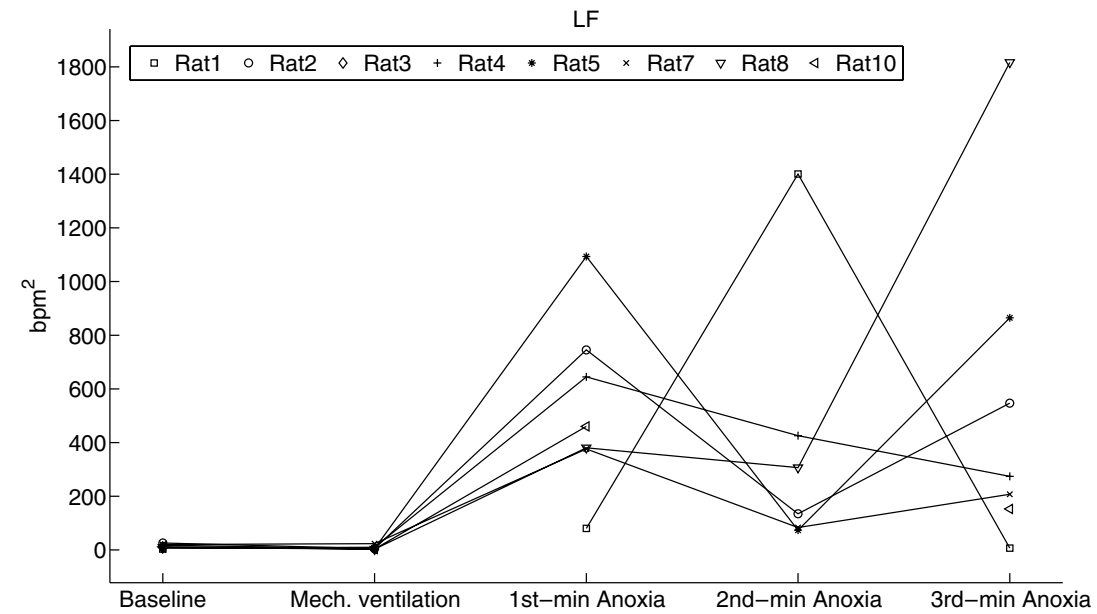

Figure 4. Low frequency index (LF) calculated for each recording, over 1-min segments without signal loss, in the sequence baseline-mechanical-ventilation-induced anoxia (first, second and third minutes).

\section{Discussion and conclusions}

Studies on combined linear and nonlinear HR analysis in rats are scarce (Moraru et al 2004, 2005, Beckers et al 2006a). This makes this subject both interesting and difficult. One such difficulty was the lack of agreement in standard frequency intervals, for HR spectral analysis, as intervals have been derived from different experimental protocols, in the absence of studies correlating them to physiological interpretation. 


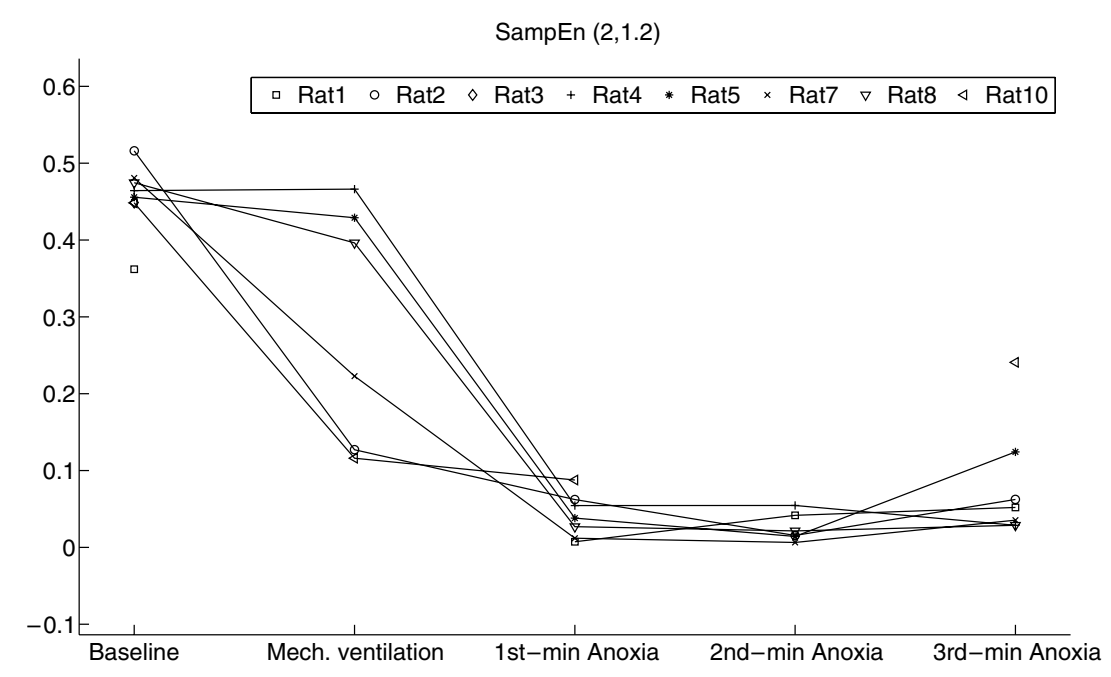

Figure 5. Sample entropy $(\operatorname{SampEn}(2,1.2))$ calculated for each recording, over 1-min segments without signal loss, in the sequence baseline-mechanical-ventilation-induced anoxia (first, second and third minutes).

In this study, linear and nonlinear HR analysis during episodes of acute anoxia in adult rats was assessed. This had to do with our particular interest in HR analysis during acute anoxia, such as that occurring in the foetus during difficult labours (Bernardes et al 1998, Gonçalves et al 2006b). Other studies have been performed before on linear and nonlinear HR analysis of acute induced anoxia in rats, but they concentrated on the evaluation of HR recovery after acute anoxia rather than on acute anoxia itself (Moraru et al 2004, 2005). This had to do with the particular interest of those authors in the study of recovery from episodes of cardiac arrest and resuscitation, which was not our case.

The values of HRV indices calculated from RR intervals are certainly different from the values of the same HRV indices calculated from HR (Niklasson et al 1993). However, in this work, we use always HRV indices calculated from the HR signal, aiming to identify pattern transitions (baseline-mechanical-ventilation-anoxia). The HR signal was chosen instead of the RR intervals in order to be coherent with the FHR signal. Therefore, although care must be taken when comparing our results with others, obtained from HRV indices calculated from RR intervals, our analysis is consistent.

The 1-min mechanical ventilation episode with room air at $1.25 \mathrm{~Hz}$ led to a clear spectral band on the HR spectrum at $1.25 \mathrm{~Hz}$. A significant decrease of mHR and of most entropy indices was also observed. However, significant differences in spectral HR indices were not found in the sequence baseline-mechanical-ventilation. An obvious explanation that arises is the fact that the referred spectral component is quite narrow (figure 2), having little contribution to the HF spectral index. This also may have been due to the lack of agreement in the standard frequency intervals considered in the analysis rather than to a lack of capability of spectral methods in the detection of spectral bands related to pathophysiological events. This aspect warrants more investigation.

The transition from the sequence baseline-mechanical-ventilation to anoxia led to a decrease of mean HR and entropy, clearly discriminating these two instances, whereas most linear indices increased, namely LF, HF and LF/HF. This denotes increased activation of 
the autonomic nervous with sympathetic imbalance and decreased or blunted activity of the HR complexity regulatory centres (Gonçalves et al 2006a, Moraru et al 2004, Beckers et al 2006b). With continued agonizing anoxia, linear indices decreased, whereas entropy remained stably low, denoting an overall decrease of the activity of all HR dynamics regulatory centres (Gonçalves et al 2006a, Moraru et al 2004, Beckers et al 2006b). All this evidence is consistent with human foetal studies performed in the final minutes of labour (Gonçalves et al 2006b, Siira et al 2005), as well as with non-human foetal studies developed during episodes of acute hypoxia or anoxia (Li et al 2004, Chaffin et al 1998).

Other HR indices could have been used, namely SD1 and SD2 indices derived from Poincaré plots (Moraru et al 2004) and scaling indices such as fractal dimension and short and long-term correlation (Beckers et al 2006a). Some of those indices, previously used by some of us in FHR analysis (Vieira et al 2004, Felgueiras et al 1998), may be useful, as the scaling behaviour of HR seems to be selectively related to the alpha-adrenergic activity whereas entropy appears to be related to cholinergic activity (Beckers et al 2006b). Those indices were outside the scope of this study, which was designed, like our last series of studies on FHR (Gonçalves et al 2006a, 2006b, 2007), to be more comparable with other FHR studies published in the literature (Magenes et al 2000, Signorini et al 2003). On the other hand, in this study an evaluation of entropy was performed in more depth than in other studies. We used entropy analysis considering different scales, something that may make a difference in FHR analysis, as suggested by studies where this was evident (Gonçalves et al 2006a).

Two major limitations of this study should be discussed. The ultimate goal of this study was trying to further elucidate the role of linear and nonlinear analysis of heart rate, in the detection and interpretation of episodes of acute anoxia-such as those occurring to the human foetus during parturition — using an adult anaesthetized rat model. First, extrapolations of observations on an anaesthetized adult rat model for non-anaesthetized human foetuses should be performed with extreme caution. Second, the same prudence should also be taken into consideration regarding the anoxia model used, particularly when it is known that this model can dramatically change the HR responses (Murasato et al 1998). These limitations do not allow full direct application of the observations of this study to foetal heart-rate monitoring. However, this study further provides clear and consistent information about the significance and possibilities of linear and nonlinear HR indices regarding the detection of pathophysiologic events. This further warrants more studies of these HR indices in rat models, in rat physiopathology studies, as well as in human studies.

In conclusion, our results highlight that the rat model is a useful model for further research and understanding of linear and nonlinear analysis of heart-rate dynamics. The transition from baseline to acute anoxia is encompassed by signs of increased activation of the autonomic nervous system with sympathetic imbalance and decreased or blunted activity of the HR complexity regulatory centres. With continued agonizing anoxia, signs of decreased activity of all $\mathrm{HR}$ regulatory centres become evident.

\section{Acknowledgments}

This study was supported by project POSI/CPS/40153/2001 from Fundação Para a Ciência e Tecnologia, Portugal.

\section{References}

ACOG 2005 American College of Obstetricians and Gynecologists. ACOG Practice Bulletin. Clinical Management Guidelines for Obstetrician-Gynecologists, Number 70, December 2005 Obstet. Gynecol. $1061453-60$ 
Ayres-de-Campos D, Bernardes J, Garrido A, Marques-de-Sá J and Pereira-Leite L 2000 SisPorto 2.0: A program for automated analysis of cardiotocograms J. Mat. Fet. Med. 9 311-8

Ayres-de-Campos D, Santos C and Bernardes J 2005 Prediction of neonatal state by computer analysis of fetal heart rate tracings: The antepartum arm of the SisPorto ${ }^{\mathbb{R}}$ validation study Eur. J. Obstet. Gynecol. Reprod. Biol. 118 52-60

Beckers F, Verheyden B and Aubert A E 2006a Aging and nonlinear heart rate control in a healthy population Am. J. Physiol. Heart Circ. Physiol. 290 H2560-70

Beckers F, Verheyden B, Ramaekers D, Swinghedauw B and Aubert A E 2006b Effects of autonomic blockade on non-linear cardiovascular variability indices in rats Clin. Exp. Pharmacol. Physiol. 33 431-9

Bernardes J, Ayres-de-Campos D, Costa-Pereira A, Pereira-Leite L and Garrido A 1998 Objective computerized fetal heart rate analysis Int. J. Gynecol. Obstet. 62 141-7

Bernardes J, Moura C, Marques-de-Sá J P and Pereira-Leite L 1991 The Porto system for automated cardiotocographic signal analysis J. Perinat. Med. 19 61-5

Cerutti C, Gustin M P, Paultre C Z, Lo M, Julien C, Vincent M and Sassard J 1991 Autonomic nervous-system and cardiovascular variability in rats-a spectral-analysis approach Am. J. Physiol. 261 H1292-9

Chaffin D G, Barnard J M, Phernetton T and Reed K L 1998 Decreased approximate entropy of heart rate variability in the hypoxic ovine fetus J. Matern. Fetal Invest. 8 23-6

Dudewicz E and Mishra S 1988 Modern Mathematical Statistics (New York: Wiley)

Felgueiras C, Marques-de-Sá J P, Bernardes J and Gama S 1998 Fractal characterisation of fetal heart rate sequences Biomedical Eng. 36 197-201

Gonçalves H, Bernardes J, Rocha A P and Ayres-de-Campos D 2007 Linear and nonlinear analysis of heart rate patterns associated with fetal behavioral states in the antepartum period Early Human Develop. 83 585-91

Gonçalves H, Rocha A P, Ayres-de-Campos D and Bernardes J 2006a Internal versus external intrapartum foetal heart rate monitoring: the effect on linear and nonlinear parameters Physiol. Meas. 27 307-19

Gonçalves H, Rocha A P, Ayres-de-Campos D and Bernardes J 2006b Linear and nonlinear fetal heart rate analysis of normal and acidemic fetuses in the minutes preceding delivery Med. Biol. Eng. Comput. 44 847-55

Hayes M 1996 Statistical Digital Signal Processing and Modelling (New York: Wiley)

Henriques-Coelho T, Correia-Pinto J, Roncon-Albuquerque R Jr, Baptista M J, Lourenco A P, Oliveira S M, BrandaoNogueira A, Teles A, Fortunato J M and Leite-Moreira A F 2004 Endogenous production of ghrelin and beneficial effects of its exogenous administration in monocrotaline-induced pulmonary hypertension Am. J. Physiol. Heart Circ. Physiol. 287 H2885-90

Kuwahara M, Yayou K, Ishii K, Hashimoto S, Tsubone H and Sugano S 1994 Power spectral analysis of heart rate variability as a new method for assessing autonomic activity in the rat J. Electrocardiology 27 333-7

Li X, Tang D, Zhou S, Zhou G, Wang C, Zhuang Y, Wu G and Shen L 2004 Redistribution of power spectrum of heart rate variability during acute umbilical artery embolism and hypoxemia in late-gestation fetal sheep Eur. $J$. Obstet. Gynecol. Reprod. Biol. 114 137-43

Lourenço A P, Roncon-Albuquerque R Jr, Bras-Silva C, Faria B, Wieland J, Henriques-Coelho T, Correia-Pinto J and Leite-Moreira A F 2006 Myocardial dysfunction and neurohumoral activation without remodeling in left ventricle of monocrotaline-induced pulmonary hypertensive rats Am. J. Physiol. Heart Circ. Physiol. 291 H1587-94

Magenes G, Signorini M G and Arduini D 2000 Classification of cardiotocographic records by neural networks IJCNN 2000: Proc. IEEE-INNS-ENNS Int. Joint Conf. on Neural Network vol 3 pp 637-41

Martinez W L and Martinez A R 2002 Computational Statistics Handbook with MATLAB (Boca Raton, FL: CRC Press)

Moraru L, Cimponeriu L, Tong S, Thakor N and Bezerianos A 2004 Characterization of heart rate variability changes following asphyxia in rats Methods Inf. Med. 43 118-21

Moraru L, Tong S, Malhotra A, Geocadin R, Thakor N and Bezerianos A 2005 Investigation of the effects of ischemic preconditioning on the HRV response to transient global ischemia using linear and nonlinear methods Med. Eng. Phys. 27 465-73

Murasato Y, Hirakawa H, Harada Y, Nakamura T and Hayashida Y 1998 Effects of systemic hypoxia on R-r interval and blood pressure variabilities in conscious rats Am. J. Physiol. 275 H797-804

Niklasson U, Wiklund U, Bjerle P and Olofsson B O 1993 Heart-rate variation-what are we measuring? Clin. Physiol. 13 71-9

Pincus S 1995 Aproximate entropy (ApEn) as a complexity measure Chaos 5 110-7

Pincus S M, Gevers E F, Robinson I C A F, VanDenBerg G, Roelfsema F, hartman M L and Veldhuis J D 1996 Females secrete growth hormone with more process irregularity than males in both humans and rats $A m$. $J$. Physiol.-Endoc. M. 270 E107-15

Pincus S and Viscarello R 1992 Approximate Entropy: a regularity measure for fetal heart rate analysis Obstet. Gynecol. 79 249-55 
Richman J S and Moorman J R 2000 Physiological time-series analysis using approximate entropy and sample entropy Am. J. Physiol. Heart Circ. Physiol. 278 H2039-49

Signorini M G, Magenes G, Cerrutti S and Arduini D 2003 Linear and nonlinear parameters for the analysis of fetal heart rate signal from cardiotacographic recordings IEEE Trans. Biomed. Eng. 50 365-73

Siira S M, Ojala T H, Vahlberg T J, Jalonen J O, Valimaki I A, Rosen K G and Ekholm EM 2005 Marked fetal acidosis and specific changes in power spectrum analysis of fetal heart rate variability recorded during the last hour of labour BJOG 112 418-23

Task force 1996 Task force of the European Society of Cardiology and the North American Society of Pacing and Electrophysiology, Heart rate variability — standards of measurement, physiological interpretation and clinical use Circulation 93 1043-65

Vieira P, Marques-de-Sá J P and Bernardes J 2004 Fractal analysis of foetal heart rate Proc. Biosignal 2004 ed J Jan, J Kozumplík and I Provazník (Brno: Vutium Press) pp 53-5 